\title{
A 2015 Medical Informatics Perspective on Health and Clinical Management: Will Cloud and Prioritization Solutions Be the Future of Health Data Management?
}

\author{
N. Bricon-Souf, E. Conchon, Section Editors for the IMIA Yearbook Section on Health and \\ Clinical Management \\ University of Toulouse, IRIT, Castres, France
}

\begin{abstract}
Summary
Objectives: Summarize current excellent research and trends in the field of Health and Clinical management.

Methods: Synopsis of the articles selected for the IMIA Yearbook 2015

Results: Three papers from international peer-reviewed journals have been selected for the Health and Clinical Management section.

Conclusion: Telemedicine is still very active in Health and clinical management, but the new tendencies on which we focus this year were firstly the introduction of cloud for health data management, with some specific security problems, and secondly an emerging expectation of prioritization tools in health care Management.
\end{abstract}

\section{Keywords}

Health and Clinical Management; Patient care management; medical record systems; technologies in healthcare; telemedicine

Yearb Med Inform 2015;10:44-6

http://dx.doi.org/10.15265//Y-2015-034

Published online August 13, 2015

\section{Introduction}

Health and Clinical Management aims at improving quality, safety and efficiency of health care. The Health and Clinical Management section, through a careful review of research papers published in 2014, highlights research trends in this area and proposes a selection of "best" papers that illustrates these new challenges.

For this yearbook edition, 1381 papers issued from the Medline bibliographic database have been considered. Last year, the Health and Clinical Management section has underlined the importance of smart and efficient exploitation of data [1]. This trend is still very important in the 2014 literature review. As in previous editions, many research works report on telemedicine trials with this year a specific focus on dermatology, chronic disease, cardiology as well as diabetes. Furthermore, two new trends can be detected: cloud based solutions for health data and prioritization of care. A general movement of moving traditional computing to the cloud actually occurs; work in conjunction with this section also follows this tendency. Cloudification of health data nevertheless challenges new issues especially in terms of confidentiality and security. Another trend that emerges concerns new ways of automatically prioritizing and distributing tasks in collaborative activities according to the context in which they occur. Feedback from datamining onto traces in complex activities are some of new performed work

\section{About the Paper Selection}

A comprehensive review was performed on the Medline database. Clinical Health Management topic directly provides terms which are rather generic, and it is not that easy to find keywords that are precise enough to focus on this theme without having a lot of noisy papers. Consequently, the performed query was built as combining some different aspects of Health and Clinical Management such as electronic record, care management or telemedicine. The query is mainly performed on title and abstract in order to be precise enough. We decided to add the idea of smart home and ambient assisting living to the previous year query, in order to be aware of some new trends for health management. The search returned a total of 1300 papers, once papers without abstract or authors were excluded. The two co-editors of the section both reviewed half of these papers and both retained about 100 papers. The selections were merged and the two co-editors reviewed independently the 200 papers. Each paper was then marked as rejected, accepted and pending. The retained papers should address a medical informatics problem and not only a clinical one. Preference was given to papers highlighting new way of addressing a problem rather than to review papers. In case of description of a study that assesses the efficiency of new proposals (a lot of papers were relating experiences with telemedicine for diabetes, for cardiology, and other chronic diseases), we tried to retain the more mature systems 
or at least the ones tested with large enough cohorts. Thirty papers were classified as kept by the two reviewers. A discussion between the two co-editors of the section leaded to a proposal of fifteen papers, a top ten selection was completed by five interesting papers which were selected as representative of the different issues emerging from the Health Clinical Management review. These 15 papers were peer-reviewed by editors and external reviewers (at least four reviewers per paper). Three papers were finally selected as best papers (Table 1). A content summary of these selected papers can be found in the appendix of this synopsis.

\section{Conclusion and Outlook}

During the review of papers from 2014 involved in Health and Clinical Management, we can notice firstly that even if a growing number of papers deals with telemedicine (lots of papers relate experiences with telemedicine applications, particularly in the chronic disease area), there is no major novelty which occurs this year in this specific domain in complement to last year review [1]. But, the integration of home centered technologies into Information Systems is still an issue as shown by Gietzelt [2]. Some propositions using new devices such as smartphones, connected devices or internet of things were also found but without noticeable improvements within the context of Health and Clinical management.

One of the main concerns developed this year is the prioritization in health. In a paper proposed by Salman[3] for example, remote triage in telehealth was proposed with prioritization processes with a particular focus on the fusion of data from multisources, another framework for prioritization was proposed by Ongenae et al.[4] providing a learning nurse call system that uses ontologies and data-mining techniques in order to regulate the nurse calls in hospital. On the topic of prioritization, we kept one of the best papers of the section entitled "developing and testing a computerized Decision Support System for Nurse-to-Patient assignment" proposed by C. Van Oosteveen et al. [5]. This paper

Table 1 Best paper selection of articles for the IMIA Yearbook of Medical Informatics 2015 in the section 'Health and Clinical Management'. The articles are listed in alphabetical order of the first author's surname.

\section{Section}

Health and Clinical Management

- Koutkias VG, McNair P, Kilintzis V, Skovhus Andersen K, Niès J, Sarfati, JC Ammenwerth E, Chazard E, Jensen S, Beuscart R, Maglaveras N. From adverse drug event detection to prevention. A novel clinical decision support framework for medication safety. Methods Inf Med 2014;53(6):482-92.

- Tong Y, Sun J, Chow SS, Li P. Cloud-assisted mobile-access of health data with privacy and auditability. IEEE J Biomed Health Inform 2014;18(2):419-29.

- Van Oostveen CJ, Braaksma A, Vermeulen H. Developing and testing a computerized decision support system for nurse-to-patient assignment: a multimethod study. Comput Inform Nursing 2014;32(6):276-85.

provides a complete discussion on the identification of relevant considerations in order to improve the nurse-to-patient assignment process. An interesting comparison between literature finding and focus group discussions allows building efficient strategies for a computerized decision support system.

Elaborating efficient framework for health and in particular introducing the use of the cloud illustrates a pretty mature topic area. A paper from Benharref et al.[6] for instance introduced the use of cloud for eHealth monitoring. The second best paper of our section [7] is concerned with the problem of privacy and auditability of healthcare data in a cloud context. The authors are Tong et al. and the paper is entitled "Cloud-Assisted Mobile-Access of Health Data with Privacy and Auditability".

The third best paper which was selected reflects the ability to build a complete process for clinical management. This paper explains how to build contextual help for clinicians through a decision support framework using knowledge extracted from actual databases thanks to data mining process. The paper is entitled "From Adverse Drug Event Detection to Prevention. A Novel Clinical Decision Support Framework for Medication Safety" from Koutkias et al [8]. The system addresses the adverse drug event prevention, and an evaluation of the proposed system is performed.

\section{Acknowledgement}

We would like to acknowledge the support of Martina Hutter and of the reviewers in the selection process of the IMIA Yearbook.

\section{References}

1. Bricon-Souf N, Conchon E. Trends on Integrating Framework of Applications or Data. Yearb Med Inform 2014;9: 55-7.

2. Gietzelt M, von Bargen T, Kohlmann M, Marschollek M, Schwartze J, Song B, et al. Home-centered health-enabling technologies and regional health information systems. An integration approach based on international standards. Methods Inf Med $2014 ; 53(3): 160-6$.

3. Salman OH, Rasid MFA, Saripan MI, Subramaniam SK. Multi-sources data fusion framework for remote triage prioritization in telehealth. J Medi Syst 2014;38(9):103.

4. Ongenae F, Claeys M, Kerckhove W, Dupont T, Verhoeve P, De Turck S. A self-learning nurse call system. Comput Biol Med 2014;44:110-23.

5. Van Oostveen CJ, Braaksma A, Vermeulen H. Developing and testing a computerized decision support system for nurse-to-patient assignment: a multimethod study. Comput Inform Nurs 2014;32(6):276-85.

6. Benharref A, Serhani MA. Novel cloud and SOAbased framework for e-health monitoring using wireless biosensors. IEEE J Biomed Health Inform 2014;18(1):46-55.

7. Tong Y, Sun J, Chow SS, Li P. Cloud-assisted mobile-access of health data with privacy and auditability. IEEE J Biomed Health Inform 2014;18(2):419-29.

8. Koutkias VG, McNair P, Kilintzis V, Skovhus Andersen K, Niès J, et al. From adverse drug event detection to prevention. A novel clinical decision support framework for medication safety. Methods Inf Med 2014;53(6):482-92.

\section{Correspondence to:}

Nathalie Souf

IRIT-Elipse

ISIS- Campus universitaire

Rue Firmin Oulès

81104 Castres

France

E-mail: nathalie.souf@irit.fr 
Appendix: Content Summaries of the Selected Best Papers for the IMIA Yearbook 2015, Section "Health and Clinical Management"

\section{Van Oostveen CJ, Braaksma A, Vermeulen H \\ Developing and testing a computerized decision support system for nurse-to- patient assignment: a multimethod study \\ Comput Inform Nursing 2014;32(6):276-85}

Preparing a fair nurse-to-patient assignment is a daily time consuming job; moreover the quality of such assignment has a true impact on nurses job satisfaction. A computerized decision support system (CDSS) is developed and tested in order to assist nursing team with such assignment process. The first step of the process was to identify relevant considerations for such assignment: a literature review helps to detect a list of such considerations and was compared to a list of considerations obtained by asking focus groups about their needs. Considerations were ranked from their importance according to the group and some differences were noticed between focus group considerations and literature ones. They were translated into constraints to feed the CDSS development based on an Integer Linear Program. The second step of work was to perform an evaluation of the proposed CDSS. Observations were performed on nurse-to-patient assignment process and results were analyzed, time reduction and lower workload were noticed after implementation of the nurse-to-patient assignment system.

\section{Tong Y, Sun J, Chow SS, Li P}

Cloud-assisted mobile-access of health data with privacy and auditability

\section{IEEE J Biomed Health Inform 2014;18(2):419-29}

In this paper, the authors present a private cloud solution for the management of health data. This private cloud provides several security key features with a focus on privacy and auditability. Indeed, electronic healthcare systems are now present in our everyday life and the amount of health data collected increase. These systems often rely on cloud based services and a large amount of personal medical data is exchanged. But, the privacy of these data is not ensured by the majority of these services. Therefore, additional solutions are needed while keeping the advantages of cloud based solutions in terms of storage capacity and computing powers. The authors propose a new architecture based on private cloud services that enforce privacy and auditability while using the infrastructure of public cloud providers such as Amazon and Google for instance. They qualitatively describe several cryptographic solutions that may be used such as Searchable Symmetric Encryption (SSE), Threshold secret sharing (TSS), Identity-Based encryption (IBE) and Attribute-Based Encryption (ABE). SSE allows the storage and indexing of encrypted documents without information leakage that can be used by an honest-but-curious party. TSS is used to share secret information among multiple entities with a distribution of the cryptographic power. IBE is an asymmetric cryptographic solution based on identity based public keys so that any party can encrypt a message with no prior key distribution. $\mathrm{ABE}$ is mainly used for fine grained access control, as every party is depicted with a set of attributes that can then be used to define an access structure to encrypted data. Only the users that own the matching attributes can the decrypt the data. Based on these four solutions, the authors proposed a novel and interesting combination that guarantee five storage requirements (data confidentiality, anonymity, unlinkability between multiple data, keyword privacy and search term privacy) as well as auditability. To address the storage privacy and the efficient retrieval of information, they choose a heuristic approach relying on SSE. While in traditional SSE, data are encrypted with the same key, they provide a new solution that enables the regular update of the key over time. They also provide a solution to hide information while preserving the searchability of the encrypted data by keywords as well as by time and date without relying on heavy cryptographic solutions. They then propose a combination of $A B E$ and Threshold signatures so that fine grained data access control (thanks to $\mathrm{ABE}$ ) and access auditability (thanks to TSS) are enforced. A performance evaluation is then made showing that the proposed solution can be deployed on mobile devices such as smartphone with a reasonable cost (16s).

\section{Koutkias VG, McNair P, Kilintzis V, Skovhus Andersen K, Niès J, Sarfati, JC Ammenwerth E, Chazard E, Jensen S, Beuscart R, Maglaveras N}

From adverse drug event detection to prevention. A novel clinical decision support framework for medication safety

\section{Methods Inf Med 2014;53(6):482-92}

The authors address the design of a decision support framework that starts to use hospital databases in order to perform data discovery on adverse drug events, employs this knowledge in order to perform a system adapted to the context of care and integrates the healthcare information technologies through the use of service-oriented architecture. The CDSS architecture is built with global management platform able to aggregate hospital data, to mine them, to deduce Cdss rules and to integrate them into a Cdss engine. A local knowledge management platform derives this knowledge on its local aspect (one of the hospital in the project). Finally a runtime platform allows contextualizing the CDSS application. The contextualization avoids over-alerting by neglecting some alerts depending on the hospital settings. A clinical validation was performed with clinician experts and test cases. The tool was implemented with the Gaston CDSS system and some specific platform management tools have been developed in java. Proofs of concepts have been developed with commercial CPOE systems. A clinical validation on about 25000 patients' records has been performed and lead to recommendations for the improvement of the CDSS. 